\title{
The role of working memory in the metaphor interference effect
}

\author{
Russell S. Pierce \\ University of California, Riverside, California \\ Rick MacLaren \\ University of Toronto at OISE \\ AND \\ Dan L. Chiappe \\ California State University, Long Beach, California
}

\begin{abstract}
Participants took longer to judge that metaphors (e.g., an insult is a razor, memory is a warehouse) were literally false than to judge that scrambled sentences (e.g., an insult is a warehouse) were false. This result is the metaphor interference effect (MIE). It demonstrates that metaphor processing is automatic. In this experiment, we found that the magnitude of the MIE is predicted by working memory (WM) capacity, with higher WM yielding a smaller MIE. This suggests that although metaphor comprehension is automatic, the early processing of metaphors is controllable by executive mechanisms. We relate our results to Kintsch's $(2000,2001)$ predication model. Specifically, we suggest that mechanisms of WM influence metaphor processing by affecting the effectiveness of the construction-integration process that identifies common properties between topics and vehicles. WM also influences the speed with which meanings are identified as literal or figurative.
\end{abstract}

The degree to which automatic behaviors can be controlled and the mechanisms that control them have long been topics of interest in cognitive psychology (J. D. Cohen, Dunbar, \& McClelland, 1990; Logan, 2002; Norman \& Shallice, 1986). A substantive theory of these mechanisms cannot be developed, however, without an account of the automatic processes being controlled (e.g., J. D. Cohen et al., 1990; Logan \& Gordon, 2001). Studies of figurative language processing have shown that metaphorical meanings are automatically activated (e.g., Glucksberg, Gildea, \& Bookin, 1982). Our goals are to demonstrate the importance of working memory (WM) as a mechanism controlling the activation of metaphorical meanings and to propose an extension of Kintsch's $(2000,2001)$ predication model as a step toward developing a more complete account of the automatic processes involved in metaphor interpretation.

The automatic activation of metaphorical meanings was first investigated by Glucksberg et al. (1982), using a task analogous to the Stroop interference task. In their experiments, people had to verify as quickly as possible whether sentences were literally true. Participants took longer to judge that metaphors (e.g., some jobs are jails, some roads are snakes) were literally false than to judge that scrambled metaphors - that is, sentences made up by combining the topics and vehicles of different metaphors (e.g., some jobs are snakes) were literally false, demonstrating that metaphorical meanings are automatically accessed. This difference in response time (RT) is the metaphor interference effect (MIE).

We predict that WM capacity will moderate the MIE. The executive component of WM is involved in ensuring that behavior is guided by goal-relevant information and in reducing the effect of irrelevant prepotent responses (Kane, Bleckley, Conway, \& Engle, 2001). For example, Kane and Engle (2003) found that high-WM individuals were less distracted by word names in the Stroop task. Similarly, in the MIE task, people must resolve the conflict in truth value arising from automatically apprehending metaphorical meanings. Success requires them to manage the interference produced by "true" metaphorical meanings when responding that the statement is literally false.

The importance of WM to metaphor production and comprehension has been previously established (Chiappe \& Chiappe, 2007; Pierce \& Chiappe, 2009). However, these studies did not assess when WM becomes involved. It is possible WM plays a role only in later stages, such as when a person is comparing alternative interpretations. In contrast, Kintsch's predication model automatically computes interpretations of metaphors by inhibiting properties associated with the vehicle that are not applicable to the

R.S.Pierce, russell.pierce@email.ucr.edu 
topic. The model thus predicts that differences in WM will affect the early stages of metaphor processing.

Although many studies have investigated the features of items that determine whether metaphorical meanings are automatically activated (e.g., Gildea \& Glucksberg, 1983; Wolff \& Gentner, 2000), few have examined the mechanisms involved therein. An exception is a study by Kazmerski, Blasko, and Dessalegn (2003) using ERP technology. They measured IQ and the N400 component, which is particularly strong when linguistic stimuli are semantically anomalous. They found that participants took longer to judge that metaphors were literally false than to judge that scrambled metaphors were and that metaphors had a smaller N400 component than did the scrambled counterparts. This shows that people process metaphors and scrambled items differently early on, with the latter indicating a greater violation of semantic expectancy. They also found that differences in the RT and amplitude of the N400 component between metaphors and scrambled items depended on IQ. The size of the MIE and N400 increased from the low-IQ to the high-IQ groups. Kazmerski et al. admitted that this effect may have been due to their use of the nonstandard RSVP presentation style, where participants are shown one word at a time and have to respond after the presentation of the final word. Those with a higher IQ may be likelier to display the MIE because they are better able to integrate the meaning of sentences under these conditions.

Kazmerski et al. (2003) assessed IQ using the K-BIT. It is important to note that the K-BIT provides measures of verbal and nonverbal intelligence, in addition to a composite IQ, but does not measure WM directly. Kazmerski et al. found that verbal intelligence predicted the magnitude of differences in the N400 component, whereas nonverbal intelligence did not. However, they did not report which type of intelligence predicts RT differences between metaphors and scrambled items. This is important because differences in N400 cannot be equated with the MIE itself, since there is no evidence that it indexes interference resolution. In short, Kazmerski et al. did not determine which mechanisms underlying intelligence are most relevant to the MIE.

Our study assessed WM and vocabulary knowledge to determine their roles in the automatic activation of metaphorical meanings. We used a standard presentation style in which participants saw entire sentences. In contrast to Kazmerski et al. (2003), we predicted that individuals with greater verbal knowledge would have a smaller MIE than those with less verbal knowledge. Specifically, individuals with greater verbal knowledge have richer and more accurate semantic networks, which include information on the literal and figurative uses of words. Networks with these properties therefore produce less of an MIE, because they allow the interference management mechanisms of WM to operate more efficiently. This prediction is consistent with evidence that measures of fluid intelligence, such as WM, are generally found to predict the ability to manage interference better than do measures of crystallized intelligence (cf. Engle, Tuholski, Laughlin, \& Conway, 1999). Conse- quently, we predicted that WM would play a stronger role in moderating the MIE than would vocabulary knowledge.

\section{METHOD}

\section{Participants}

One hundred fifty-three people participated. Of these, we dropped 9 from analysis, either due to equipment malfunction or because they did not properly complete all the tasks. The analyses were conducted on the remaining 144 participants (114 female and 30 male; $M$ age $=19.91$ years, range $=18-40$ years $)$.

\section{Materials and Procedure}

Materials for this experiment included PCs equipped with the laboratory software Superlab 4 and button boxes to record responses. The participants completed tasks in the following order: (1) word span task (WSPAN), (2) metaphor interference (MIE) task, and (3) Peabody Picture Vocabulary Task (PPVT III).

WSPAN. To assess WM, the participants saw sets of words on a computer screen, one at a time, and at the end they had to report the words in the set. Sets ranged from two to six words. To calculate the WSPAN score, we summed the number of words recalled on perfectly recalled trials. The highest possible score was 60 . The mean WSPAN score was $27.65(S D=9.58$, range $=12-60)$. Although this is a simple span task, as compared with other tasks (e.g., Engle et al., 1999), it does require managing proactive interference (Lustig, May, \& Hasher, 2001). Specifically, doing well requires participants to suppress words from earlier sets so that they do not interfere with memory for the current set. This was important for our study because measures of proactive interference predict the ability to suppress unwanted thoughts (Verwoerd, Wessel, \& de Jong, 2009).

MIE task. The participants saw sentences of the form Some $X$ are $Y$ and had to respond as quickly as possible whether they were literally true. We measured RTs from the onset of the stimulus to the onset of the response. They saw three sets of sentences: one practice set and two experimental sets. The practice set consisted of 48 items randomly arranged but constrained such that no item type appeared more than three times in a row. The practice set contained eight metaphors (e.g., some lectures are lullabies), eight scrambled metaphors (e.g., some lectures are jails), eight anomalous sentences (e.g., some spices are bees), and 24 true statements (e.g., some weather is snow). We gave feedback to the participants such that they had to correct their answers before proceeding to the next item. There were two experimental lists, each containing two sets of items. Each set contained 48 items arranged in the same proportions as those used in the practice list, but we provided no feedback. We borrowed items for this experiment with permission from Wolff and Gentner (2000) and from Glucksberg et al. (1982). These are available on request. Prior to each sentence, a fixation point was shown (i.e., ${ }^{* * * * * * * * * *}$ ). The participants had to press the leftmost button on a response box to make it disappear. Once they did so, a sentence appeared in the center of the screen. It stayed on the screen until the participants responded by pressing either the leftmost button (labeled "true") or the second from the left button (labeled "false"). The mean RT for metaphor and scrambled items correctly responded to within $4,000 \mathrm{msec}$ was $1,500 \mathrm{msec}$ $(S D=598 \mathrm{msec})$. See Table 1 for RT and accuracy for each sentence type. Although our participants committed more errors on literally true items than on literally false items, this pattern is consistent with other studies of the MIE (e.g., Kazmerski et al., 2003; Wolff \& Gentner, 2000) and did not directly influence our statistical analyses.

PPVT III. This test assesses receptive vocabulary knowledge (Dunn \& Dunn, 1997). The participants were presented with a word aurally. Then they were shown four pictures and were asked to click the picture that best illustrated the meaning of the word. The participants were assigned a score based on the total number of words they correctly recognized. The mean PPVT score was 169.48 ( $S D=$ 11.51 , range $=126-191)$. 
Table 1

Response Times and Accuracy Rates

\begin{tabular}{cccc}
\multicolumn{4}{c}{ Response Times and Accuracy Rates } \\
\hline Sentence Type & $\begin{array}{c}\text { Correct } \\
\text { Response } \\
\text { Times }(\mathrm{msec})\end{array}$ & $\begin{array}{c}\text { Incorrect } \\
\text { Response } \\
\text { Times }(\mathrm{msec})\end{array}$ & $\begin{array}{c}\text { Accuracy } \\
(\%)\end{array}$ \\
\hline Metaphor & 1,538 & 1,750 & 91.45 \\
Scrambled & 1,466 & 1,773 & 97.18 \\
Anomalous & 1,391 & 1,572 & 97.40 \\
True & 1,379 & 1,692 & 80.00 \\
\hline
\end{tabular}

Note-All response times in excess of 4,000 msec were removed from these means and from analysis.

\section{Analytical Technique}

The present study used linear mixed effects regression, rather than an ANOVA (Baayen, Davidson, \& Bates, 2008). Others have used linear mixed effects regression in similar situations (e.g., Kliegl, Risse, \& Laubrock, 2007). It has the advantage of being able to consider continuous (e.g., WM and vocabulary knowledge) and categorical (e.g., metaphor vs. scrambled items) variables simultaneously without losing power or increasing Type I error rate, due to aggregation of data within arbitrarily specified cells (J. Cohen, Cohen, West, \& Aiken, 2003). In addition, this analytical technique allowed us to simultaneously control for differences in participant and item response latency (or accuracy), instead of performing separate participant and item analyses or computing $F^{\prime}$ min.

All analyses were conducted using the $\mathrm{R}$ statistical program (R Development Core Team, 2008) with the models fit using the lmer function of the lme4 package (Bates, 2007) evaluated on the basis of $\chi^{2}$ goodness-of-fit tests or the AIC, as appropriate (Baayen et al., 2008). Details regarding the structure of the model are available from the first author. In addition, we conducted correlational analyses between the MIE and various participant factors to make our results clear to those unfamiliar with our primary statistical analysis method.

\section{RESULTS AND DISCUSSION}

\section{Sentence Falsification Error Rate}

Controlling for response speed, we tested whether there was a difference in the probability that a participant would mistakenly respond true when an item was a metaphor versus when an item was a scrambled metaphor. The participants were $157 \%$ more likely to correctly reject scrambled items than metaphorical items $(Z=2.44, p=.01)$. This result is what one would expect if metaphor processing is an automatic process that can interfere with judging the literal falsehood of a statement. Consistent with the notion of a speed-accuracy trade-off, we found that responses made rapidly $(-1 S D)$ were $32 \%$ more likely to be in error than were those made more slowly $(+1 S D)$ $(Z=3.77, p<.001)$.

\section{MIE Magnitude}

We evaluated whether there was a difference in RTs to falsify scrambled statements versus metaphors. The difference between these conditions is the MIE. On average, across metaphors and scrambled items, the participants responded after $1,524 \mathrm{msec}(S E=46 \mathrm{msec})$, but they were $90 \mathrm{msec}(S E=45 \mathrm{msec})$ faster in responding to scrambled items than to metaphors $(Z=2.00, p<.05)$. The magnitude of this MIE, although noticeably larger than the 11- to 35-msec MIE observed by Kazmerski et al. (2003), is comparable to that observed by Glucksberg et al. (1982) and Wolff and Gentner (2000).

\section{Vocabulary Knowledge}

To identify the role of vocabulary knowledge in falsification times, we extended the previous model to include vocabulary knowledge. Those with high vocabulary scores $(+1 S D)$ responded to items $191 \mathrm{msec}(S E=62 \mathrm{msec})$ faster than did those with low vocabulary scores $(-1 S D)$ $(Z=3.08, p=.002)$. The MIE for high-vocabulary participants $(+1 S D)$ was $63 \mathrm{msec}$, whereas the MIE for low-vocabulary participants $(-1 S D)$ was $118 \mathrm{msec}$; this interaction was only marginally significant $(p=.07)$. However, the raw correlation between the modeled MIE and vocabulary knowledge was significant $(r=-.26$, $p=.001)$.

These results show that greater vocabulary knowledge makes people faster at rejecting metaphors and scrambled items. In addition, although only marginally significant, we observed a trend for those with more vocabulary knowledge to have less of an MIE than did those with less vocabulary knowledge. This contrasts with the results of Kazmerski et al. (2003), who found that only people with higher verbal knowledge displayed the MIE. This suggests that their findings may indeed have been an artifact of their use of the RSVP presentation technique.

\section{Working Memory}

To identify the role of WM in falsification times, we extended the model identified while investigating vocabulary scores to include WSPAN. We removed the interaction between the MIE and vocabulary knowledge from further analyses for three reasons. First, when appropriate statistical methods were employed, it did not reach conventional levels of significance. Second, a partial correlation between the MIE and PPVT scores, controlling for WSPAN scores, was also not significant $(r=.16, p=.06)$. Third, we observed a correlation between PPVT and WSPAN scores $(r=.35, p<.001)$. Consequently, models including both interactions would suffer from excessive multicollinearity (J. Cohen et al., 2003). Given the complexity of our statistical model, our already large sample size, and the theoretical importance of WM, model respecification was selected as an appropriate solution.

We found that, after controlling for the main effect of vocabulary knowledge, the participants with high WSPAN scores $(+1 S D)$ responded to items $232 \mathrm{msec}(S E=$ $62 \mathrm{msec}$ ) faster than did those with low WSPAN scores $(-1 S D)(Z=3.65, p<.001)$. There was also an interaction between the magnitude of the MIE and WM $(Z=$ $2.19, p=.03)$. The MIE for high-WSPAN participants $(+1 S D)$ was $58 \mathrm{msec}$, whereas the MIE for low-WSPAN participants $(-1 S D)$ was $123 \mathrm{msec}$. The raw correlation between the MIE and WSPAN was likewise significant $(r=-.36, p<.001)$ and remained significant in a partial correlation controlling for vocabulary knowledge $(r=$ $-.29, p<.001)$. This contrasts with the partial correlation between vocabulary knowledge and the MIE, which was only marginally significant after controlling for WSPAN. 
To rule out the possibility, suggested by a reviewer, that the effect of WM was simply a consequence of faster participants' showing smaller effects of everything, we extended the error model to include $\mathrm{WM}$ as a predictor. Although, overall, responses made more rapidly are more likely to be in error than those made more slowly, we found that those participants with high WM were $32 \%$ more likely to select a correct response than were those with low working memory $(Z=2.44, p=.01)$. Therefore, there is something qualitatively different occurring in the sentence processing of individuals as a function of WM, beyond simple changes in processing speed. They are better at managing the interference posed by metaphors.

\section{Theoretical Implications}

Evidently, having higher WM allows people to be faster at judging metaphors and scrambled items to be literally false. Furthermore, as was predicted, people with higher WM were less distracted by metaphorical meanings. Our results add to the literature showing that WM plays an important role in the suppression of irrelevant prepotent responses (e.g., Conway, Cowan, \& Bunting, 2001; Kane et al., 2001).

The results of our study can be explained using Kintsch's $(2000,2001)$ predication model, a computational account of the controlled and automatic processes involved in understanding literal and figurative predication statements (i.e., statements of the form $A$ is a $P$ ). The model uses two components: latent semantic analysis (LSA) and construction-integration (CI). LSA represents the meaning of words as vectors indicating their position in a 300-dimension semantic space. Semantic similarity between words is indicated by the cosine of their vectors, and words close to each other in this space are in the same semantic neighborhood. People with greater vocabulary knowledge are likelier to represent this information accurately and so will be better able to process statements efficiently. The CI component uses these LSA vectors to construct the interpretation of statements.

To process statements, the model activates the $m$ closest terms in the semantic neighborhood of the vehicle. These terms indicate important features associated with words and the contexts in which they are used. As Kintsch (2001) says,

For most sentences combining familiar terms in expected ways [i.e., literal statements], $m=20$ works well, because terms related to A will be found even among the closest neighbors of P. For metaphors ... where the predicate and argument can be quite distant, the crucial terms are usually not found among the top 100 neighbors, and $m$ needs to be set larger, say 500 neighbors. (p. 180)

Once activated, those terms in the neighborhood of the vehicle and also in the neighborhood of the topic are used to modify the vehicle's vector. This modifies its meaning to make it appropriate to the context of use. This is carried out by spreading activation through an inhibitory network. The network consists of the topic, vehicle, and the $m$ clos- est members of the vehicle's semantic neighborhood. After several rounds of spreading activation, the network settles. Those properties in the neighborhood of the vehicle, but not in that of the topic, are inhibited, whereas those appearing in both remain active. Among those, the $k$ properties with the greatest activation represent the meaning.

An omission in Kintsch's model is an account of how $m$ is set appropriately. Control of $m$ entirely through WM is implausible. If metaphor processing involved deliberate adaptation of the $m$ parameter, participants would typically need low values of $m$ and would know to adjust to a higher value of $m$ only after literal processing had failed. As is demonstrated by the MIE, literal processing does not have priority over metaphor processing. We propose that when semantic expectancies are violated, $m$ may automatically increase to widen the scope of processing and that this increase in $m$ may be correlated with a greater N400. Some evidence in support of this proposal may already exist. Kazmerski et al. (2003) found that those with a smaller N400 experienced less of an MIE. In addition, Lai, Curran, and Menn (2009) recently found that metaphorical and anomalous sentences exhibited a greater N400 than did literally true sentences; this similarity between metaphorical and anomalous sentences may reflect a consistency of cognitive processing related to the differences in $m$ parameter settings congruent with our proposal.

Kintsch's predication model can also be used to explain the role of WM in metaphor comprehension. Specifically, WM might affect metaphor processing through the operation of the CI component. Those with high WM are more accurate and rapid in their ability to inhibit irrelevant links during CI. Consequently, CI will require fewer rounds of spreading activation to settle, allowing high-WM individuals to arrive at better interpretations faster than low-WM individuals. In the context of the MIE task, participants will reject scrambled items as false when no common properties are identified between topics and vehicles. For the metaphors, however, interpretations will be found, and participants have to determine whether they are literal. WM may play a role here as well. One possibility is that the judgment of whether a statement is literal or figurative may depend on the ratio of the summed activations of the $k$ properties over $m$. When vehicles are literally true of topics, this ratio is high, because $m$ is low and activated properties of the vehicle fit well with the topic. When vehicles are figuratively true, this ratio is low because $m$ will be much higher. With greater WM capacity, such a check is likely to be more accurate and faster, yielding a smaller MIE.

To conclude, this study shows that although metaphor processing is automatic, some aspects are controllable by WM. In terms of Kintsch's predication model, we suggest that metaphor processing is triggered automatically by violations of semantic expectancies that cause people to consider a wider semantic neighborhood. WM is crucial, however, in moderating the MIE and, thus, the early processing of metaphors. The exact role of WM remains a subject for future research, but we suggest two possibilities. WM may speed the process through which metaphorical meanings are identified in the construction-integration 
stage, by efficiently suppressing irrelevant vehicle properties. WM may also enable people to identify the literal or figurative status of statements more efficiently.

\section{AUTHOR NOTE}

We appreciate the work and time of all of our research assistants, especially our lead research assistants, Wadad Itani and Henry Cuevas. We thank Timothy Gann and Dale Barr for providing sound statistical advice. Correspondence regarding this article should be addressed to R. S. Pierce, Department of Psychology, University of California, Riverside, 900 University Ave., Riverside, CA 92521 (e-mail: russell.pierce@, email.ucr.edu)

\section{REFERENCES}

BaAyen, R. H., Davidson, D. J., \& Bates, D. M. (2008). Mixed-effects modeling with crossed random effects. Journal of Memory \& Language, 59, 413-425. doi:10.1016/j.jml.2007.12.005

BATES, R. H. (2007). Lmer4: Linear mixed-effects models using S4 classes (R package version 0.99875-7). Retrieved from http://cran.r -project.org/src/contrib/Archive/lme4/lme4_0.99875-7.tar.gz

ChiAPPE, D. L., \& ChIAPPE, P. (2007). The role of working memory in metaphor production and comprehension. Journal of Memory \& Language, 56, 172-188. doi:10.1016/j.jml.2006.11.006

Cohen, J., Cohen, P., West, S. G., \& Aiken, L. S. (2003). Applied multiple regression/correlation analysis for the behavioral sciences (3rd ed.). Mahwah, NJ: Erlbaum.

Cohen, J. D., Dunbar, K., \& McClelland, J. L. (1990). On the control of automatic processes: A parallel distributed processing account of the Stroop effect. Psychological Review, 97, 332-361. doi:10.1037/0033-295X.97.3.332

Conway, A. R. A., Cowan, N., \& Bunting, M. F. (2001). The cocktail party phenomenon revisited: The importance of working memory capacity. Psychonomic Bulletin \& Review, 8, 331-335.

Dunn, L., \& Dunn, M. (1997). Peabody Picture Vocabulary Test-III. Circle Pines, MN: American Guidance Services.

Engle, R. W., Tuholski, S. W., Laughlin, J. E., \& Conway, A. R. A. (1999). Working memory, short-term memory, and general fluid intelligence: A latent-variable approach. Journal of Experimental Psychology: General, 128, 309-331. doi:10.1037/0096-3445.128.3.309

GildEA, P., \& GLUCKSBERG, S. (1983). On understanding metaphor: The role of context. Journal of Verbal Learning \& Verbal Behavior, 22. 577-590. doi:10.1016/S0022-5371(83)90355-9

Glucksberg, S., Gildea, P., \& Bookin, H. B. (1982). On understanding nonliteral speech: Can people ignore metaphors? Journal of Verbal Learning \& Verbal Behavior, 21, 85-98. doi:10.1016/S0022 $-5371(82) 90467-4$

Kane, M. J., Bleckley, M. K., Conway,A. R. A., \& Engle, R. W. (2001). A controlled-attention view of working-memory capacity. Journal of
Experimental Psychology: General, 130, 169-183. doi:10.1037/0096 $-3445.130 .2 .169$

KANe, M. J., \& ENGLE, R. W. (2003). Working-memory capacity and the control of attention: The contributions of goal neglect, response competition, and task set to Stroop interference. Journal of Experimental Psychology: General, 132, 47-70. doi:10.1037/0096-3445.132.1.47

Kazmerski, V. A., Blasko, D. G., \& Dessalegn, B. G. (2003). ERP and behavioral evidence of individual differences in metaphor comprehension. Memory \& Cognition, 31, 673-689.

KINTSCH, W. (2000). Metaphor comprehension: A computational theory. Psychonomic Bulletin \& Review, 7, 257-266.

KINTSCH, W. (2001). Predication. Cognitive Science, 25, 173-202. doi:10 .1016/S0364-0213(01)00034-9

Kliegl, R., Risse, S., \& Laubrock, J. (2007). Preview benefit and parafoveal-on-foveal effects from word $n+2$. Journal of Experimental Psychology: Human Perception \& Performance, 33, 1250-1255. doi:10.1037/0096-1523.33.5.1250

Lai, V. T., Curran, T., \& Menn, L. (2009). Comprehending conventional and novel metaphors: An ERP study. Brain Research, 1284, 145-155. doi:10.1016/j.brainres.2009.05.088

LoGAN, G. D. (2002). An instance theory of attention and memory. Psychological Review, 109, 376-400. doi:10.1037/0033-295X 109.2.376

LogAN, G. D., \& Gordon, R. D. (2001). Executive control of visual attention in dual-task situations. Psychological Review, 108, 393-434 doi: $10.1037 / 0033-295 X .108 .2 .393$

Lustig, C., May, C. P., \& Hasher, L. (2001). Working memory span and the role of proactive interference. Journal of Experimental Psychology: General, 130, 199-207. doi:10.1037/0096-3445.130.2.199

Norman, D. A., \& Shallice, T. (1986). Attention to action: Willed and automatic control of behavior. In R. T. Davidson, G. E. Schwartz, \& D. Shapiro (Eds.), Consciousness and self-regulation: Advances in research and theory (pp. 1-18). New York: Plenum.

Pierce, R. S., \& ChiaPPE, D. L. (2009). The roles of aptness, conventionality and working memory in the production of metaphors and similes. Metaphor \& Symbol, 24, 1-19. doi:10.1080/10926480802568422

R Development Core Team (2008). R: A language and environment for statistical computing. Vienna: R Foundation for Statistical Computing.

Verwoerd, J., Wessel, I., \& DE Jong, P. J. (2009). Individual differences in experiencing intrusive memories: The role of the ability to resist proactive interference. Journal of Behavior Therapy \& Experimental Psychiatry, 40, 189-201. doi:10.1016/j.jbtep.2008.08.002

WolfF, P., \& Gentner, D. (2000). Evidence for role-neutral initial processing of metaphors. Journal of Experimental Psychology: Learning, Memory, \& Cognition, 26, 529-541. doi:10.1037/0278 $-7393.26 .2 .529$

(Manuscript received August 5, 2009; revision accepted for publication December 22, 2009.) 